\title{
Tratamento terapêutico multi-profissional para endometriose com dor pélvica
}

\author{
Raquel Pinto Caldeira ${ }^{1}$ \\ Vanessa Mara Alves Noronha ${ }^{2}$ \\ Bruna Eschiletti Corrêa de Oliveira ${ }^{3}$ \\ Fleurismar Gonçalves Portela de Amorim ${ }^{4}$
}

\section{Resumo}

O objetivo do trabalho foi especificar os vários tipos de tratamentos de endometriose voltados à finalidade de melhoria da dor pélvica presente. Acerca da realização de pesquisa bibliográfica com artigos relacionados à dor pélvica e endometriose, foram encontrados 65 artigos, excluídos 49, restando apenas 16 . Concluiu-se que o uso de qualquer hormônio é eficaz no tratamento clínico medicamentoso para dor pélvica associada à endometriose. Dependendo da gravidade da doença encontrada, a prática ideal da laparoscopia consiste em realizar o diagnóstico e a extinção cirúrgica dos focos de endometriose no ato operatório. A toda paciente deve ser indicado tratamento multiprofissional que atenda às demandas físicas e psicológicas dela, visando ao alívio da dor, à melhora da qualidade de vida e, principalmente, de sua auto-estima.

Palavras-chave: Endometriose. Dor pélvica. Tratamento.

\section{Introdução}

A endometriose é uma doença que afeta a mulher em idade reprodutiva, sendo caracterizada por implante e crescimento de tecido endometrial (glândulas e/ou estroma) fora da cavidade uterina. Tem sido observada em 5

\footnotetext{
${ }^{1}$ Estudante do curso de fisioterapia no Uniceub. Email: quelcaldeira@hotmail.com

${ }^{2}$ Estudante do curso de fisioterapia no Uniceub. Email: vmaranoronha@yahoo.com

${ }^{3}$ Estudante do curso de fisioterapia no Uniceub. Email: bruninhaecorrea@hotmail.com

${ }^{4}$ Estudante do curso de fisioterapia no Uniceub. Email: fleurisportella@yahoo.com.br
} 
a $10 \%$ das pacientes submetidas a laparotomias ginecológicas, 20 a $50 \%$ das mulheres com infertilidade e 60 a $70 \%$ das portadoras de dor pélvica crônica. Em mulheres assintomáticas, a incidência é de 1 a $2 \%$. A incidência média da doença é bastante discutida; acredita-se, atualmente, que atinge em torno de $10 \%$ da população feminina em idade reprodutiva (LORENÇATTO et al., 2002; MOURA et al., 1999; NAVARRO et al., 2006; PETTA et al., 2005; MARQUES et al., 2004; LORENÇATTO et al., 2006).

Dor pélvica crônica é definida como dor pélvica não menstrual ou não cíclica, com duração de pelo menos seis meses, suficientemente intensa para interferir em atividades habituais, implicando necessidade de tratamento clínico ou cirúrgico. Essa é uma queixa habitual na clinica ginecológica, e as terapias médicas atuais, freqüentemente, não resolvem. A prevalência estimada de dor pélvica crônica é de 3,8\% em mulheres de 15 a 73 anos (superior à enxaqueca, asma e dor nas costas) (LORENÇATTO et al., 2002; NOGUEIRA et al., 2006; BERNARDES; BAHAMONTES, 2005; MODOTTE; DIAS, 2000; HOWARD, 2003).

Existe, enfim, uma relação entre endometriose e dor pélvica, pois a incidência de dor pélvica em pacientes com endometriose é muito alta. A dor pélvica é a queixa mais freqüente em pacientes que procuram a ginecologia; assim, pode ser que tenham endometriose.

Esta revisão tem por objetivo especificar os vários tipos de tratamentos de endometriose, com a finalidade de propiciar melhora para pacientes com dor pélvica.

\section{Metodologia}

A revisão foi realizada por meio de pesquisa nas bases de dados Pubmed e Scielo, durante o período de 16 de março de 2008 a 22 de maio de 2008. Para a busca dos artigos nas bases, foram utilizadas as palavras-chave "dor pélvica" e "endometriose", e como critério de escolha estabeleceu-se que o artigo deveria fazer referência à relação entre endometriose e dor pélvica. 


\section{Resultados}

Foram encontrados 65 artigos. O primeiro critério de exclusão foi retirar os artigos que não falavam de dor pélvica, já que a maioria dos trabalhos encontrados só discorria sobre endometriose. Após essa primeira seleção, foram excluídos 32 artigos. Como segundo critério de exclusão, foram indicados os trabalhos em que a dor pélvica não tinha relação com endometriose restando, assim, 17 artigos. Depois, restaram apenas 16 trabalhos, com os quais foi realizada a revisão.

\section{Endometriose}

Endometriose é uma doença crônica, atualmente ainda sem cura definitiva, mas com diversos tratamentos. Seu diagnóstico é feito por meio da história clínica, exame físico e exames complementares. Uma das possibilidades de exame físico é a palpação vaginal. Dentre os exames complementares, pode ser realizada ultra-sonografia com transdutores vaginais, dopplervelocimetria colorida, ultrasonografia transvaginal tridimensional, tomografia computadorizada, ressonância nuclear magnética, laparoscopia e dosagens de marcadores como o CA-125, proteína $\mathrm{C}$ reativa e anticorpos anticardiolipina. O quadro clínico é variável, sendo a dor pélvica, a dispareunia e a infertilidade a tríade típica mais freqüentemente encontrada (MOURA et al., 1999; NOGUEIRA et al., 2006).

Há discordância quanto ao uso de exames invasivos para o diagnóstico de endometriose com dor pélvica, porque nem todos os pacientes submetidos a esses exames saem com o diagnóstico confirmado, ainda que $30 \%$ a $80 \%$ dos pacientes submetidos a esse tipo de exame apresentem anormalidades. Entretanto, há estudos mostrando que procedimentos cirúrgicos podem minimizar de $40 \%$ para $5 \%$ casos com dor pélvica e devem ser precedidos da avaliação clínica, laboratorial e de imagem cuidadosa (NOGUEIRA et al., 2006).

Petta et al. (2007), em um estudo, concluíram que, quando uma paciente se queixa de infertilidade, dismenorréia ou dor pélvica, os médicos suspeitam de endometriose para o diagnóstico, e que ginecologistas mais informados sobre o assunto suspeitam de endometriose precocemente. 
O uso de terapia medicamentosa para endometriose é baseado no fato de que a endometriose responde a hormônios. Duas condições fisiológicas, gravidez e menopausa, estão freqüentemente associadas à resolução da dor provocada pela endometriose. O tratamento hormonal visa a inibir a produção de gonadotrofinas, anulando a esteroidogênese e, subseqüentemente, a liquefazer, necrosar e absorver os implantes. $\mathrm{O}$ tratamento pode ser realizado com a administração contínua de anticoncepcionais orais (ACO), progestagênio sintético ou agonistas do GnRH (gonadotropin-reasing hormone). Além disso, para resolução da infertilidade associada, as pacientes podem ser submetidas a técnicas de reprodução assistida como a inseminação intra-uterina (IUI) ou fertilização in vitro (FIV), esta última com resultados mais promissores (MOURA et al., 1999; NAVARRO et al., 2006).

O tratamento da endometriose deve ser individualizado, considerando sempre os sintomas e o impacto da doença sobre a qualidade de vida da paciente. Uma equipe multidisciplinar especializada deve ser (sempre que possível) envolvida, na tentativa de fornecer um tratamento capaz de abranger todos os aspectos biopsicossocial da paciente (NAVARRO et al., 2006).

\section{Dor pélvica}

A dor pélvica pode ser leve ou intensa, e não existe relação dessa com a gravidade da endometriose. Mas a dor pélvica não é, apenas, relacionada à endometriose, tem-se um diagnóstico fechado para ela: dor pélvica não menstrual ou não cíclica, com duração de pelo menos seis meses. Isto é, mesmo que não se saiba a causa da dor, pode-se fechar o diagnostico de dor pélvica (NOGUEIRA et al., 2006; HOWARD, 2003).

A etiologia da dor pélvica crônica não é clara e, usualmente, resulta de uma complexa interação entre os sistemas gastrintestinal, urinário, ginecológico, músculo-esquelético, neurológico, psicológico e endócrino, influenciado ainda por fatores socioculturais. Entretanto, quando um diagnóstico específico pode ser feito, o tratamento também é especifico para essa patologia; quando não, o tratamento deve ser dirigido para o controle da dor (NOGUEIRA et al., 2006; HOWARD, 2003). 
Um fator importante do diagnóstico de dor pélvica é a realização de uma boa anamnese e exame físico. A anamnese deve ser minuciosa e, nela, deve-se questionar sobre as características da dor, localização, fatores de melhora e piora, duração, intensidade e sua relação com o ciclo menstrual. Deve-se dar atenção, também, à história obstétrica (número e tipo de partos), cirurgias pélvicas anteriores e tratamentos já realizados. Durante o exame físico geral, especial atenção deve ser dada ao modo de andar, características faciais de sofrimento e posturas antálgicas, a palpação deve ser realizada da maneira mais delicada possível e confortável, pois a sensibilidade dolorosa dessas pacientes está, freqüentemente, exacerbada (NOGUEIRA et al., 2006; HOWARD, 2003).

\section{Fisiopatologia da dor pélvica}

Atualmente, têm-se enfatizado o papel da inflamação neurogênica na fisiopatologia da dor pélvica crônica. Para embasar essa hipótese, parte-se do princípio, universalmente aceito, de que estímulos nocivos, por dano tecidual, podem aumentar a produção de substâncias promotoras de dor que estão presentes nas terminações dos nociceptores aferentes primários e são liberadas quando o nociceptor é estimulado. Por outro lado, quando uma fibra sensitiva é estimulada eletricamente, o impulso caminha não só em direção à medula espinhal (sentido ortodrômico), mas, também, no sentido inverso, para a periferia (sentido anti-drômico). Quando esse estímulo anti-drômico chega à periferia, há liberação de óxido nítrico, substância P, proteína relacionada ao gene da calcitonina (CGRP), neuroquinina $\mathrm{A}$ e $\mathrm{B}$, dentre outros, que causam a inflamação neurogênica, caracterizada por vasodilatação, edema e hiperalgesia. Isso gera mais lesão tecidual, o que fecha o ciclo e faz o estímulo doloroso se perpetuar. Esse mecanismo permite interpretar que a dor pélvica tem, ao menos em parte, um componente inflamatório crônico importante (NOGUEIRA et al., 2006).

Para médicos que atendem mulheres com o diagnóstico de dor pélvica, é importante compreender, ao menos em parte, os tipos de dor. Isso pode facilitar a elaboração da hipótese diagnóstica. Resumidamente, têm-se:

1- Dor de origem somática: o estímulo doloroso inicia em estruturas como pele, músculos, fáscias, ossos e articulações. Freqüentemente, é menos intensa, geralmente em pontadas, 
e a paciente, em geral, consegue localizar um ponto específico de dor;

2- $\quad$ Dor de origem visceral: usualmente é mal localizada, por ter sensibilidade cruzada entre vísceras que compartilham uma mesma inervação. Freqüentemente em cólicas, às vezes associadas a fenômenos autonômicos, como náuseas, vômitos e reações emocionais;

3- Dor de origem psicológica: embora alterações de personalidade, de conduta e depressão tenham papel bem definido na maneira de percepção da dor, a dor pélvica crônica psicogênica é menos freqüente e é diagnóstico de exclusão (NOGUEIRA et al., 2006; WENOF; PERRY, 1999).

\section{Tratamento clínico de endometriose com dor pélvica}

O tratamento clínico medicamentoso para dor pélvica associada à endometriose é altamente eficaz, com taxas de sucesso que variam de 80 a 100\% de melhora e um intervalo livre dos sintomas que pode chegar a dois anos (NAVARRO et al., 2006).

As drogas hormonais investigadas - acetato de medroxiprogesterona (AMP), gestrinona, Contraceptivos Orais Combinados (COC), elcometrina, danazol e agonistas do GnRH mostram-se igualmente efetivas no alívio da dor. Contudo, os efeitos adversos apresentados e os custos são diferenciados e devem ser levados em consideração quando da escolha terapêutica (NAVARRO et al., 2006; NOGUEIRA et al., 2006; HOWARD, 2003; DULEBA et al., 1996).

Os prostágenos são uma forma de progesterona sintética, entretanto, ao invés de causar alterações secretoras no endométrio durante a fase lútea do ciclo menstrual normal, eles causam atrofia endometrial por diminuição da síntese de receptores de estrogênio. $\mathrm{O}$ acetato de metroxiprogesterona (ADM) é um dos progestágenos mais usados, mas devem ser levados em conta os efeitos colaterais (acne, dor localizada e vasodilatação) apresentados em $40 \%$ das pacientes que usaram $50 \mathrm{mg} /$ dia . Já gestrinona é um progestágeno com atividade androgênica e, quando comparada com o agonistas do GnRh, parece ter efeito semelhante no controle da dor e na satisfação dos pacientes. Porém, essa medicação (200 a 300mg/ 
semana) apresenta efeitos colaterais que, muitas vezes, não são suportadas pelas pacientes, como amenorréia, spotting (manchas), acne, hirsutismo, edema e ganho de peso, que pode chegar até a $3 \mathrm{Kg}$ em seis meses de tratamento (NAVARRO et al., 2006; HOWARD, 2003; DULEBA et al., 1996).

Elcometrina é um contraceptivo que não tem ação por via oral. Contudo, se administrado por via subdérmica, sua ação é de progestágeno que não interfere na produção normal de estrogênio orgânico. No estudo, a elcometrina apresenta resultados melhores que qualquer outro tipo de hormônio, pois seus efeitos colaterais são mínimos, sendo os principais a diminuição da libido e a dor em membros inferiores, levando-se em conta que $29 \%$ não apresentavam queixas (COUTINHO et al., 1999).

Os contraceptivos orais combinados (COC) são a primeira escolha para tratamento clínico em muitos centros, por ser este um tratamento simples, pouco dispendioso, de fácil manejo e com bons resultados embasados pela literatura. Porém, ainda não está claro o mecanismo de ação sobre os focos de endometriose desse regime de tratamento. Mas é interessante notar e considerar que, em pacientes cujo principal sintoma é a dismenorréia, o uso continuo de COC leva à amenorréia, promovendo melhores resultados a curto prazo no controle da dismenorréia e na melhora da qualidade de vida das pacientes (NAVARRO et al., 2006.; HOWARD, 2003; DULEBA et al., 1996).

A supressão ovariana e, conseqüentemente, da menstruação é tratamento eficiente para o controle da dor pélvica crônica de várias etiologias. Pode ser realizada com contraceptivos hormonais combinados, progestágenos ou agonistas do GnRH em pacientes principalmente com componente cíclico e dismenorréia, por períodos determinados. Além da endometriose com dor pélvica crônica, várias alterações podem beneficiar-se desse tratamento, entre elas, síndrome da congestão pélvica, patologias uterinas sintomáticas como miomas e adenomiose, cistite intersticial e síndrome do intestino irritável (NOGUEIRA et al., 2006; HOWARD, 2003; DULEBA et al., 1996).

O danazol é um androgênio que suprime as gonadotrofinas e age inibindo a ovulação. A posologia adequada é de $600 \mathrm{mg} /$ dia. Porém, a incidência dos efeitos colaterais é muito alta, atingindo cerca de $85 \%$ das usuárias. Os principais 
efeitos adversos descritos são: ganho de peso, edema, diminuição no tamanho das mamas, acne, hirsutismo, oleosidade na pele e alterações no timbre da voz, além da potencial influência negativa sobre o metabolismo dos lipídeos, com elevação dos níveis de LDL-colesterol e colesterol total (NAVARRO et al., 2006; HOWARD, 2003; DULEBA et al., 1996).

Agonistas do GnRH são considerados, hoje, o tratamento padrão contra dor associada à endometriose, devido ao estado de hipoestrogenismo que acarretam. A média de tempo para recidiva dos sintomas de dismenorréia, dispareunia e dor pélvica acíclica é muito variável: entre 6 e 12 meses após o final do tratamento. Porém, geralmente a dor é de menor intensidade. Alguns autores preconizam seu uso por seis meses, outros por três meses, mas a tendência parece ser de utilizá-los pelo menor tempo necessário. O grande inconveniente para o uso do agonista do GnRH no tratamento da dor associada à endometriose certamente é a ocorrência de efeitos adversos secundários ao hipoestrogenismo, como ondas de calor, ressecamento vaginal, cefaléia, tontura, labilidade emocional, acne, mialgia, edema, redução no volume mamário, ganho de peso, diminuição na libido, insônia, perda de massa óssea (NAVARRO et al., 2006; HOWARD, 2003; DULEBA et al., 1996)

Petta et al. (2005) realizaram um estudo randomizado com um total de 82 mulheres com endometriose, dismenorréia ou dor pélvica crônica, dividas em 2 grupos. Um grupo teve o tratamento com levonorgestrel intra-uterino, e o outro, com análogos de GnRH durante 6 meses, sendo avaliados, diariamente, a dor e o sangramento e, uma vez por mês, a qualidade de vida. Não houve diferença entre os grupos quanto à diminuição da dor, que foi significante. $\mathrm{O}$ grupo que usava levonorgestrel teve sangramento maior do que o que usava agonista do GnRH. Também não houve diferença quanto à qualidade de vida. Foram efetivos os dois tratamentos para alívio de dor, devendo-se levar em consideração que o levonorgestrel pode ser usado por até 5 anos.

O tratamento pós-operatório com danazol ou agonista do $\mathrm{GnRH}$ por seis meses reduz a dor associada à endometriose e posterga a recorrência da doença. Contudo, o tratamento pós-operatório com COC não é tão efetivo (NAVARRO et al., 2006). 


\section{Tratamento cirúrgico da endometriose com dor pélvica}

A vídeo-laparoscopia possibilita uma visão direta da cavidade pélvica e abdominal e é útil para estabelecer o diagnóstico definitivo ou, pelo menos, para excluir a presença de patologias pélvicas (MODOTTE; DIAS, 2000).

Dependendo da gravidade da doença encontrada, a prática ideal consiste em realizar o diagnóstico e a extinção cirúrgica dos focos de endometriose no mesmo ato operatório. Assim, recomenda-se obter o consentimento livre e esclarecido no pré-operatório, abrangendo caracterização do procedimento que poderá ser realizado, complicações possíveis e taxas de sucesso. Não há dados concretos que justifiquem a prescrição de tratamento hormonal antes da cirurgia, para melhorar as taxas de sucesso na abordagem cirúrgica (NAVARRO et al., 2006).

Recomenda-se que, em procedimento laparoscópico para diagnóstico, ao se encontrar foco de endometriose, seja realizado o procedimento necessário (debridamento da área), evitando-se, assim, outra intervenção cirúrgica. A eficiência do tratamento depende da retirada completa da lesão. Deve-se ter cuidado na retirada dos focos de endometriose, pois pode haver algumas complicações em lesões múltiplas e profundas, com maior incidência (FAHMY et al., 2005; DULEBA et al., 1996).

Tanto a destruição das lesões por coagulação, fulguração ou vaporização quanto a extinção das lesões superficiais mostram resultados semelhantes relativos à melhora na dor pélvica associada à endometriose (NAVARRO et al., 2006; DULEBA et al., 1996).

Quanto à abordagem dos endometriomas ovarianos em pacientes portadoras de dor pélvica, a discussão principal é sobre a retirada total da pseudocápsula ou sua drenagem e coagulação. A literatura mostra uma tendência à técnica de drenagem e coagulação, que também permite obter material para análise anatomopatológica (NAVARRO et al., 2006).

Nos casos em que há endometriose infiltrativa profunda, seja de septo retovaginal, intestinal ou vesical, o benefício do tratamento cirúrgico é muito grande. A laparoscopia pode ser realizada com sucesso para abordagem da endometriose do septo reto-vaginal, proporcionando grande melhora na dor pélvica crônica e cursando com baixas taxas de complicação. Assim, podemos concluir que o tratamento cirúrgico propicia melhora clínica significativa dos sintomas ginecológicos e intestinais que 
acometem mulheres com esse tipo de endometriose. Tal tratamento deve ser sempre encorajado, porém, realizado por cirurgião experiente, e a paciente deve estar ciente das possíveis complicações (NAVARRO et al., 2006; DULEBA et al., 1996).

Modotte e Dias (2000) realizaram um estudo prospectivo incluindo $32 \mathrm{mu}-$ lheres portadoras de dor pélvica, que foram submetidas à videolaparoscopia diagnóstica. A média de idade das pacientes do estudo foi de 30 anos, variando de um mínimo de 16 anos a um máximo de 41 anos. Foram encontradas aderências pélvicas em $28,1 \%$ das pacientes examinadas, endometriose em $34,4 \%$, e $12,5 \%$ apresentavam varizes pélvicas. Em $25 \%$ das pacientes, o exame foi normal.

Pode ser feito ainda um tratamento de neurectomia que consiste na transsecção ou extinção dos nervos, pela injeção de produtos químicos neurotóxicos ou por cirurgia que visa a usar energia suficiente pra destruir o tecido neural (calor, frio, laser). Em pacientes com dor pélvica associada à endometriose com queixa de dismenorréia severa, realiza-se a extinção do plexo hipogástrico superior (nervo présacral) (HOWARD, 2003; DULEBA et al., 1996).

Quanto à retirada de ligamentos uterossacros e neurectomia pré-sacral, embora haja melhora transitória da dor em alguns casos, existem poucas evidências de melhora à longo prazo. Esse procedimento tem sido adotado, com melhor eficácia, em casos de dor pélvica crônica decorrente de endometriose severa associada à dismenorréia (NOGUEIRA et al., 2006; HOWARD, 2003).

\section{Tratamento multiprofissional}

O tratamento de endometriose tem como principal objetivo o alívio das queixas e dos sintomas, ou seja, busca-se o alívio da dor pélvica e a recuperação da fertilidade por meio de medicação e/ou cirurgia. Contudo, muitas mulheres apresentam queixa de dor após se submeterem a diversas intervenções, o que gera desgaste emocional, altera seu rendimento profissional, sua relação familiar e afetiva e reduz sua qualidade de vida e, principalmente, sua auto-estima. Logo, deve-se buscar um tratamento multiprofissional que atenda às demandas físicas e psicológicas das mulheres com endometriose, objetivando o alívio da dor e o retorno da paciente para a atividade funcional e vida familiar (LORENÇATTO et al., 2007; BERNARDES; BAHAMONTES, 2005) 
Com base em estudo realizado com 60 mulheres com diagnóstico de endometriose, por meio do questionário SF-36, para avaliar a qualidade de vida, Marques et al. (2004) concluíram que elas têm pobre qualidade de vida, independentemente da intensidade da dor, do uso de medicamentos ou da convivência com um parceiro. Mulheres com endometriose moderada ou severa tiveram melhor pontuação nos aspectos emocionais do questionário. Entre as mulheres que se exercitavam regularmente, foi observada melhor pontuação na saúde mental.

A avaliação psicológica ajuda a fornecer ao médico, informações específicas da paciente, as quais poderão ser usadas no planejamento do tratamento. Pessoas com problemas psicossociais, tais como abuso sexual, discórdia matrimonial, distúrbios de personalidade, problemas familiares, podem ser mais vulneráveis às sensações dolorosas que acompanham todo o processo da doença. No entanto, ainda não foi comprovada a relação entre o perfil psicológico e a intensidade da dor relatada pela paciente (LORENÇATTO et al., 2002; NOGUEIRA et al., 2006; HOWARD, 2003).

Lorençatto et al. (2002), em um estudo com 50 pacientes, com o objetivo de avaliar a freqüência de depressão em mulheres portadoras de endometriose e com queixa de dor pélvica, constataram que $92 \%$ das entrevistadas tinham depressão em intensidades variadas. A presença desta pode dificultar ou mesmo impedir o tratamento da dor pélvica em pacientes com endometriose.

Lorençatto et al. (2006) realizaram, também, um estudo cross-sectional e prospectivo, comparando a prevalência de depressão em mulheres com endometriose. Foi analisada a evolução de 100 mulheres com endometriose, divididas, igualmente, em 2 grupos, um com dor pélvica crônica e outro livre de dor, aplicando-se o questionário de depressão de Beck. Concluiu-se que a depressão tem alta prevalência em mulheres com endometriose, especialmente naquelas com dor pélvica. A avaliação cuidadosa pode identificar mulheres com depressão, as quais podem beneficiar-se com o tratamento.

Pacientes com dor pélvica geralmente são ansiosas e apresentam depressão associada, o que aumenta, ainda mais, a complexidade dessa síndrome. O humor pode ficar comprometido, interferindo na interpretação e no relato da dor. Elas apresentam, também, graves rupturas na situação ocupacional, familiar e social, com conseqüente impacto na qualidade de vida. A relação entre essas duas condições é 
controversa: tanto a depressão quanto a dor pélvica crônica são formas comuns de somatização; e, assim como a dor pode ser uma expressão somática do desconforto psicológico, a depressão pode ser uma resposta à dor pélvica (MODOTTE; DIAS, 2000; LORENÇATTO et al., 2002)

O tratamento psicológico é importante, pois a depressão e a somatização alteram a percepção da dor; entretanto, não se pode esquecer dos aspectos físicos da dor. A dor pode ser controlada com a aplicação de estimulação elétrica nervosa transcutânea (TENS), que age por meio de dois mecanismos: opióides endógenos e teoria das comportas. Estimulação elétrica é um recurso terapêutico freqüentemente usado no alívio da dor por não apresentar efeitos colaterais, ser barato, não invasivo e de aplicação simples. Por isso, deve ser considerada a melhor opção terapêutica para melhorar a qualidade de vida de mulheres com dor pélvica (BERNARDES; BAHAMONTES, 2005; LORENÇATTO et al., 2007; DULEBA et al., 1996).

Bernardes \& Bahamontes (2005) fizeram um estudo com um total de $24 \mathrm{mu}-$ lheres que tinham dor pélvica crônica por, no mínimo, 6 meses. Elas passaram por 10 sessões de estimulação elétrica intra-vaginal, com freqüência de $8 \mathrm{~Hz}$, largura de pulso de $1 \mathrm{msec}$ e intensidade ajustada de acordo com a sensação da paciente. $\mathrm{O}$ tratamento consistiu em 30 minutos por aplicação, de 2 a 3 vezes por semana. Avaliada a evolução da dor na segunda semana, na quarta semana e no sétimo mês após o termino do tratamento, ela se mostrou significantemente menor. Conclui-se, então, que o tratamento foi efetivo.

Após estudo realizado por Lorençatto et al. (2007) com 128 mulheres, distribuídas, igualmente, em dois grupos - um recebeu intervenção em grupo e outro não -, foi identificada diminuição significativa dos escores de dor e de depressão ao final da intervenção em grupo. As mulheres que não participaram dos grupos de apoio mostraram escores maiores de dor quando comparados aos escores iniciais das participantes dos grupos. Já os escores de depressão foram menores no grupo sem intervenção. A intervenção de grupo foi eficaz na diminuição da dor e da depressão das mulheres com endometriose.

Existem ainda algumas formas de terapias alternativas que podem ser usadas no alívio da dor, como: acupuntura, reflexologia, hipnose e biofeedback. Acupuntura consiste na aplicação de estímulos sobre a pele. Agulhas são colocadas em locais 
específicos à profundidade exata; as agulhas podem ser manipuladas, aquecidas ou permanecer no local por um longo tempo. Hipnose, reflexologia e biofeedback são todas tentativas de controlar a dor ao nível cerebral da interpretação dos estímulos nocivos. Essas técnicas parecem ser muito eficazes em alguns indivíduos (DULEBA et al., 1996).

\section{Conclusão}

Existem muitas formas de se tratar endometriose. Todavia, quando ela está relacionada com dor pélvica, há tratamento mais específico, podendo ser clínico medicamentoso, com o uso, em geral, de drogas hormonais do tipo: acetato de medroxiprogesterona (AMP), gestrinona, contraceptivos orais combinados (COC), elcometrina, danazol e agonista do GnRH. Em relação à sua eficácia, tais medicamentos são igualmente efetivos no alívio da dor. Entretanto, devem ser levados em consideração os efeitos colaterais causados por cada um.

Já o tratamento cirúrgico depende da gravidade da doença. Ele é indicado para o tratamento de endometrioses mais leves. Se realizada laparoscopia diagnóstica, caso sejam encontrados endometriomas, deve ser feito o debridamento da área, aproveitando-se a incisão cirúrgica.

Deve-se dar importância, também, ao tratamento multiprofissional, com relação à terapia psicológica, à terapia com TENS e, ainda, à terapia alternativa. Recomendamos que haja maior indicação desses tratamentos por parte dos médicos e que, assim, seu uso cresça.

\section{Therapeutic multi-professional treatment for endometriosis with pelvic pain}

\section{Abstract}

The purpose of the work was to comment about the several types of treatment for endometriosis to improve the pelvic pain presented. We accomplished a bibliographic review with papers related to pelvic pain and endometriosis, and were found 65 articles, excluded 49 , leaving only 16 . We concluded that the use 
of any hormone is effective in the clinic medical treatment for pelvic pain associated to the endometriosis. Depending on the severity of the disease found, the better practice of the laparoscopy is to perform the diagnosis and surgical extinction of the endometriosis's focal points in surgery. Every patient should be given to the multiprofessional treatment that addressed the physical and psychological demands aimed at the relief of pain, improves quality of life and especially their self-esteem.

Keywords: Endometriosis. Treatment. Pelvic pain.

\section{Referências}

BERNARDES, N. O.; BAHAMONTES, L. Intravaginal electrical stimulation for the treatment of cronic pelvic pain. The Journal of Reproductive Medicine, [S.l], v. 50, n. 4, p .267-272, 2005.

COUTINHO, E. M. et al. Tratamento de endometriomas ovarianos com implantes subcutâneos de ST-1435 (Elcometrina). Revista Brasileira de Ginecologia e Obstetrícia., Rio de Janeiro, v. 21, n. 10, p. 597-602, 1999.

DULEBA, A. J.; KELTZ, M. D; OLIVE, D. L. Evaluation and management of chronic pelvic pain. The Journal of the American Association of Gynecologic Laparoscopists. [S.1], v.3, n.2, 205-227, 1996.

FAHMY, W. M. et al. Avaliação dos resultados do tratamento cirúrgico de pacientes portadoras de endometriose do septo retovaginal. Revista Brasileira de Ginecologia e Obstetrícia, Rio de Janeiro, v. 27, n. 10, p. 613-618, 2005.

HOWARD, F. M. Chronic pelvic pain. The American College of Obstetricians and Gynecologists, Nova York, v. 101, n. 3, 2003.

LORENÇATTO, C. et al. Depression in women with endometriosis with and without chronic pelvic pain. Acta Obstetrician et Gynecologica Scandinavica, [S.l], v. 85, n. 1, p. 88-92. 2006.

LORENÇATTO, C. et al. Avaliação de dor e depressão em mulheres com endometriose após intervenção multiprofissional em grupo. Revista da Associação Médica Brasileira, São Paulo, v. 53, n. 5, p .344-348, 2007. 
LORENÇATTO, C. et al. Avaliação da freqüência de depressão em pacientes com endometriose e dor pélvica. Revista da Associação Médica Brasileira, São Paulo, v. 48 , n. 3, p.217-221, 2002.

MARQUES, A. et al. Quality of Life in Brazilian Women with Endometriosis Assessed Through a Medical Outcome Questionnaire. The Journal of Reproductive Medicine, [S.1], v. 49, n. 2, p .115-120, 2004.

MODOTTE, W. P.; DIAS, R. Utilização da Minilaparoscopia em pacientes com Algia Pélvica. Revista Brasileira de Ginecologia e Obstetrícia, Rio de Janeiro, v. 22, n .2, p. 95-100, 2000.

MOURA, M. D. et al. Avaliação do tratamento clínico da endometriose. Revista Brasileira de Ginecologia e Obstetrícia, Rio de Janeiro, v. 21, n. 2, p. 85-90, 1999.

NAVARRO, P. A. A. S., BARCELOS, I. D. S. R.; SILVA, J. C. Tratamento da endometriose. Revista Brasileira de Ginecologia e Obstetrícia, Rio de Janeiro, v. 28, n .10, p .612-623, 2006.

NOGUEIRA, A. A.; REIS, F. J. C.; POLI NETO, O. B. Abordagem da dor pélvica crônica em mulheres Revista Brasileira de Ginecologia e Obstetrícia, Rio de Janeiro, v. 28, n. 12, p. 733-740, 2006.

PETTA, C. A. et al. Randomized clinical trial of a levonorgestrel-releasing intrauterine system and a depot GnRH analogue for the treatment of chronic pelvic pain in women with endometriosis. Human Reproduction, [S.l], v.20, n.7, p. 1993-1998, 2005.

PETTA, C. A. et al. Current practice in the management of symptoms of endometriosis: A survey of brazilian gynecologists. Revista da Associação Médica Brasileira, São Paulo, v. 53, n. 6, p.525-529, 2007.

WENOF, M.; PERRY, C. P. Chronic pelvic pain: a pacient education booklet. Chronic Pelvic Pain. Disponível em: <http://www.pelvicpain.org $>$. Acesso em: 26 set. 2007. 